\title{
Photovoice: A Novel Approach to Improving Antituberculosis Treatment Adherence in Pune, India
}

\author{
Sangita C. Shelke, ${ }^{1}$ Prakash S. Adhav, ${ }^{1}$ Patrick K. Moonan, ${ }^{2}$ Matthew Willis, ${ }^{2}$ \\ Malan A. Parande, ${ }^{1}$ Srinath Satyanarayana, ${ }^{3}$ Vikas D. Kshirsagar, ${ }^{1}$ and Smita Ghosh ${ }^{2}$ \\ ${ }^{1}$ Department of Preventive and Social Medicine, Byramjee Jeejeebhoy Government Medical College, Pune, India \\ ${ }^{2}$ Division of Tuberculosis Elimination, Centers for Disease Control and Prevention, Atlanta, GA, USA \\ ${ }^{3}$ International Union Against Tuberculosis and Lung Disease, South-East Asia Regional Office, New Delhi, India
}

Correspondence should be addressed to Smita Ghosh; gpr4@cdc.gov

Received 23 May 2014; Accepted 11 September 2014; Published 7 October 2014

Academic Editor: Paul R. Klatser

Copyright (C) 2014 Sangita C. Shelke et al. This is an open access article distributed under the Creative Commons Attribution License, which permits unrestricted use, distribution, and reproduction in any medium, provided the original work is properly cited.

\begin{abstract}
We compared antituberculosis treatment (ATT) adherence and outcomes among patients exposed to Photovoice (video of previously cured TB patients sharing experiences about TB treatment) versus those not exposed. The odds of successful outcome (i.e., cured or completing treatment) for the 135 patients who watched Photovoice were 3 times greater (odds ratio: 2.8; 95\% CI: 1.3-6.1) than for patients who did not watch Photovoice. The comparison group, on average, missed more doses (10.9 doses; $95 \%$ CI: 6.6-11.1) than the intervention group who saw Photovoice (5.5 doses; 95\% CI: 3.7-6.1). Using Photovoice at initiation of ATT has the potential to improve treatment adherence and outcomes.
\end{abstract}

\section{Introduction}

Tuberculosis (TB) requires successful completion of at least six months of antituberculosis treatment (ATT) [1]. Unfortunately, ATT compliance is difficult. Long treatment duration, common side effects of the medication, and other problems such as poverty and unemployment have been reported as potential barriers to ATT adherence [2]. ATT nonadherence leads to prolonged infectiousness, acquisition of drug resistance, relapse, lower cure rates, and higher mortality rates $[3,4]$. In 2012, more than a third of the states in India reported that $>20 \%$ of patients either lost to follow-up or were not cured at the conclusion of ATT [5], suggesting that a substantial proportion of patients are nonadherent to ATT.

ATT adherence often depends upon the patients' general knowledge of TB, socioeconomic status, and whether they believe in the efficacy of the medication $[6,7]$. Photovoice is an anthropologic storytelling method that allows participants to use mixed media to record and share personal experience to influence behavioural change and promote public health action [8]. In our study, we used video recordings to capture the personal experiences about tuberculosis treatment and treatment adherence of previously treated tuberculosis patients. We sought to determine if watching a Photovoice video before ATT would reduce the number of missed doses and improve treatment outcomes.

\section{Methods}

The study was conducted at BJGMC, Sassoon General Hospital, Government TB Treatment Centre. BJGMC treats approximately 4,000 tuberculosis patients per year and offers medical service to over 9 million people of the Pune, the second most populous district in Maharashtra state [9]. Utilizing the Photovoice approach [7], a 15-minute video was produced in Marathi, the local language spoken in Maharashtra. The video production, costing approximately $\$ 500$, included eight previously cured patients expressing their personal stories about TB, their attitudes, perceptions and beliefs about ATT, and how ATT had a positive effect on their well-being and recovery from illness. All patients emphasized the benefits of treatment adherence and importance of completing ATT. We approached every third newly registered patient at BJGMC 
TABLE 1: Demographic, risk, and treatment outcomes of TB patients who viewed the Photovoice video and comparison group who did not, Pune, India.

\begin{tabular}{|c|c|c|c|c|c|c|}
\hline \multirow{2}{*}{ Characteristics } & \multicolumn{2}{|c|}{ Photovoice } & \multicolumn{2}{|c|}{ Comparison } & \multirow{2}{*}{ Total } & \multirow{2}{*}{$P$ value } \\
\hline & $n$ & $(\%)$ & $n$ & $(\%)$ & & \\
\hline \multicolumn{7}{|l|}{ Gender } \\
\hline Male & 87 & $(64.4)$ & 77 & $(54.6)$ & 164 & \multirow{2}{*}{0.096} \\
\hline Female & 48 & $(35.6)$ & 64 & $(45.4)$ & 112 & \\
\hline \multicolumn{7}{|l|}{ Age categories (years) } \\
\hline$<15$ & 17 & $(12.6)$ & 18 & $(12.8)$ & 35 & \multirow{6}{*}{0.148} \\
\hline $15-25$ & 23 & $(17.0)$ & 30 & $(21.3)$ & 53 & \\
\hline $26-35$ & 33 & $(24.4)$ & 42 & $(29.8)$ & 75 & \\
\hline $36-45$ & 31 & $(23.0)$ & 33 & $(23.4)$ & 64 & \\
\hline $46-55$ & 23 & $(17.0)$ & 9 & $(6.4)$ & 32 & \\
\hline$>55$ & 8 & $(5.9)$ & 9 & (6.4) & 17 & \\
\hline \multicolumn{7}{|l|}{ HIV status } \\
\hline Positive & 45 & $(33.3)$ & 41 & $(29.1)$ & 86 & \multirow{3}{*}{0.743} \\
\hline Negative & 66 & $(48.9)$ & 74 & $(52.5)$ & 140 & \\
\hline Unknown & 24 & $(17.8)$ & 26 & $(18.4)$ & 50 & \\
\hline \multicolumn{7}{|l|}{ Type of tuberculosis } \\
\hline Sputum positive pulmonary & 56 & $(41.5)$ & 52 & $(36.9)$ & 108 & \multirow{3}{*}{0.423} \\
\hline Sputum negative pulmonary & 31 & $(22.9)$ & 28 & $(19.9)$ & 59 & \\
\hline Extrapulmonary & 48 & $(35.6)$ & 61 & $(43.3)$ & 109 & \\
\hline \multicolumn{7}{|l|}{ Total number of missed doses } \\
\hline No missed doses & 102 & $(75.6)$ & 66 & $(46.8)$ & 168 & \multirow{2}{*}{0.000} \\
\hline 1 or more missed doses & 33 & $(24.4)$ & 75 & $(53.2)$ & 108 & \\
\hline \multicolumn{7}{|c|}{ Sputum at the start of initiation phase ${ }^{1}$} \\
\hline Negative & 81 & $(60.0)$ & 95 & $(67.4)$ & 176 & \multirow{2}{*}{0.125} \\
\hline Positive & 54 & $(40.0)$ & 46 & $(32.6)$ & 100 & \\
\hline \multicolumn{7}{|c|}{ Sputum at the end of initiation phase ${ }^{1}$} \\
\hline Negative & 133 & $(98.5)$ & 124 & $(87.9)$ & 257 & \multirow{2}{*}{0.000} \\
\hline Positive & 2 & $(1.5)$ & 17 & $(12.1)$ & 19 & \\
\hline \multicolumn{7}{|l|}{ Treatment outcomes } \\
\hline Successful & 125 & $(92.6)$ & 115 & $(81.6)$ & 240 & \multirow{2}{*}{0.000} \\
\hline Unsuccessful $^{2}$ & 10 & $(7.4)$ & 26 & $(18.4)$ & 36 & \\
\hline
\end{tabular}

${ }^{1}$ Antituberculosis treatment (ATT) is comprised of 2 phases: intensive phase (IP) (24 doses of isoniazid, rifampicin, pyrazinamide, and ethambutol by directly observed therapy (DOT) thrice a week on alternate days for 8 weeks) and continuation phase (CP) (54 doses of isoniazid and rifampicin given thrice a week on alternate days with at least first dose of every week being directly observed). ${ }^{2}$ Patients with treatment failure and patients who transferred, lost to follow-up, or died.

during January 1, 2011, to March 31, 2011, to participate in the study. After giving written informed consent, patients were shown the Photovoice video and initiated ATT as per national guidelines [5]. Every third treatment card of newly registered patient during January 1, 2010, to March 31, 2010, was reviewed as a comparison group. These patients had not viewed the Photovoice video. No other interaction with study investigators occurred after the start of treatment for both groups. New TB patients were defined as persons not previously treated for TB and in the first month of ATT. Previously treated patients were not approached to enroll nor were they included in the comparison group. After TB diagnosis all patients were referred to a Directly Observed Treatment Strategy (DOTS) centre located nearest to their residence. Each DOTS centre administers ATT and maintains a TB treatment card documenting the number of missed doses during TB treatment and treatment outcomes upon completion.

Demographic and clinical variables, number of doses prescribed, number of doses taken during treatment, and treatment outcomes were collected from the TB treatment cards of patients in both the Photovoice and comparison groups. Treatment outcomes were defined as successful or unsuccessful according to national treatment guidelines described elsewhere [5]. Successful treatment outcome included patients who were considered cured or who completed a full course of ATT. Unsuccessful treatment outcome included patients who failed treatment, transferred, lost to follow-up, or died during ATT [5].

Pearson chi-square and independent sample $t$-tests were used to compare differences in proportions between successful and unsuccessful outcome among the Photovoice and 
comparison groups. Odds ratios (OR) were used to measure the association of Photovoice with sputum status at the end of the intensive phase (IP) and with treatment outcomes. All statistical tests were considered to be significant at an alpha of $<0.05$.

All patients were treated according to national guidelines irrespective of their participation or refusal to take part in the study. All data were safeguarded to protect patient confidentiality and no individual patient identifiers were retained electronically. The study protocol was approved by the International Union Against TB and Lung Disease (Paris, France) and the Institutional Review Board of BJGMC.

\section{Results}

Among 144 patients selected to view the Photovoice video, 135 (94\%) consented to participate. In total, 276 patients (Photovoice group $n=135$; comparison group $n=141$ ) were studied. There were no significant differences in age, HIV status, or clinical presentation between Photovoice and comparison groups (Table 1). Almost two-thirds (76\%) of the patients who watched the Photovoice video did not miss any doses compared to $47 \%$ among the comparison group (Table 1). A larger proportion of patients in the Photovoice group had successful treatment outcomes compared to the comparison group (93\% versus $82 \%$ ) (Table 1 ). The Photovoice group had three times the odds of successful treatment outcome compared to those who did not watch Photovoice (OR: 2.8, 95\% CI: 1.3-6.1) (Table 1).

Nearly all (99\%) of the Photovoice patients converted sputum at the end of IP compared to $88 \%$ in the comparison group (Table 1). Patients who did not watch the video, on average, missed a greater number of doses during treatment (10.9 doses; 95\% CI: 6.6-11.1) than patients in the intervention group (5.5 doses; 95\% CI: 3.7-6.1) (Table 2).

\section{Discussion}

Several studies have described factors associated with ATT adherence but few studies describe the impact of interventions leading to behavioral changes that influence treatment outcomes [6]. This study was not a rigorous randomized controlled trial; however, these results reflect meaningful programmatic experience. Improved TB health-related education, along with efforts to reduce stigma, has been shown to improve patient health-seeking behaviours, lower treatment default rates, and bring about greater treatment completion $[7,9]$. To our knowledge, this is the first time that Photovoice has been used to empower TB patients to share their personal experiences to influence ATT adherence. Having patients watch a 15-minute Photovoice video prior to initiation of ATT is a low-resource activity with the potential to improve medication adherence and long-term treatment outcomes.

\section{Conclusion}

Photovoice is a simple, inexpensive strategy that might be considered as one of Government of India's Information, Education, and Communications activities to strengthen TB
TABLE 2: Mean number of antituberculosis drug doses missed among TB patients who viewed Photovoice and comparison group who did not by treatment outcome.

\begin{tabular}{|c|c|c|c|}
\hline & $N(\%)$ & $\begin{array}{c}\text { Mean number } \\
\text { of total missed } \\
\text { doses } \\
\text { Mean (SD) }\end{array}$ & $\begin{array}{c}\text { Mean difference } \\
(95 \% \mathrm{CI})^{2} \\
\end{array}$ \\
\hline \multicolumn{4}{|l|}{ Overall $(N=276)$} \\
\hline \multicolumn{4}{|l|}{ Photovoice } \\
\hline Successful & $125(92.6)$ & $0.6(1.3)$ & \multirow{2}{*}{$4.9(3.7-6.1)$} \\
\hline Unsuccessful $^{3}$ & $10(7.4)$ & $5.5(4.9)$ & \\
\hline \multicolumn{4}{|l|}{ Comparison } \\
\hline Successful & 115 (81.6) & $2.1(3.9)$ & \multirow{2}{*}{$8.8(6.6-11.1)$} \\
\hline Unsuccessful $^{3}$ & $26(18.4)$ & $10.9(8.6)$ & \\
\hline
\end{tabular}

treatment adherence. In combination with other interventions, a Photovoice approach could be an effective tool for reduction of treatment lost to follow-up and other unsuccessful TB treatment outcomes.

\section{Disclaimer}

The findings and conclusions in this paper are those of the authors and do not necessarily represent views of the Centers for Disease.

\section{Conflict of Interests}

The authors declare that there is no conflict of interests regarding the publication of this paper.

\section{Acknowledgments}

The authors acknowledge the RNTCP Programme staff, Photovoice Advisory Committee members, the cured TB patients who worked on production of the Photovoice video, Dr. Pradeep Awate, and Mr Nagesh and his team for their relentless efforts in the making of the video and without their support this study would not be possible. The study was conducted as part of the "TB Operations Research Training Project" which aimed to build operational research capacity within the Government of India's Revised National Tuberculosis Control Programme (RNTCP). This training project was conceived and implemented jointly by the Central Tuberculosis Division (Directorate General of Health Services, Ministry of Health and Family Welfare, Government of India), the National Tuberculosis Institute (Directorate General of Health Services, Ministry of Health and Family Welfare, Government of India Bangalore, India), the World Health Organization (WHO, India Country Office), the International Union Against Tuberculosis and Lung Disease (The Union, South-East Asia Regional Office, New Delhi, India), and the US Centers for Disease Control and Prevention (CDC; Division of Tuberculosis Elimination, Atlanta, USA). 


\section{References}

[1] World Health Organization, Guidelines for Treatment of Tuberculosis, WHO/HTM/TB/2010.05, WHO, Geneva, Switzerland, 4th edition, 2010.

[2] T. R. Frieden and J. A. Sbarbarob, "Promoting adherence to treatment for tuberculosis: the importance of direct observation," Bulletin of the World Health Organization, vol. 85, no. 5, pp. 407-409, 2007.

[3] E. Sumartjo, "When tuberculosis treatment fails. A social behavioral account of patient adherence," The American Review of Respiratory Disease, vol. 147, no. 5, pp. 1311-1320, 1993.

[4] World Heath Organization, "Adherence to long-term therapies: evidence for Action," in Tuberculosis, chapter 15, p. 145, World Heath Organization, Geneva, Switzerland, 2003, http://www .who.int/chp/knowledge/publications/adherence_section3.pdf.

[5] Central TB Division, Annual Status Report, 2012, http://tbcindia.nic.in/pdfs/TB\%20India\%202012-\%20Annual\%20Report .pdf.

[6] J. Volmink and P. Garner, "Interventions for promoting adherence to tuberculosis management," Cochrane Database of Systematic Reviews, no. 4, Article ID CD000010, 2000.

[7] S. Vijay, P. Kumar, L. S. Chauhan, B. H. Vollepore, U. P. Kizhakkethil, and S. G. Rao, "Risk factors associated with default among new smear positive TB patients treated under DOTS in India," PLoS ONE, vol. 5, no. 4, Article ID e10043, 2010.

[8] C. Wang and M. A. Burris, "Photovoice: concept, methodology, and use for participatory needs assessment," Health Education and Behavior, vol. 24, no. 3, pp. 369-387, 1997.

[9] “BJ Government Medical College," http://www.bjmc.org/. 


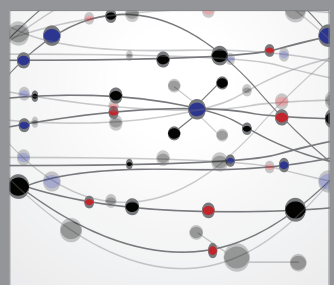

The Scientific World Journal
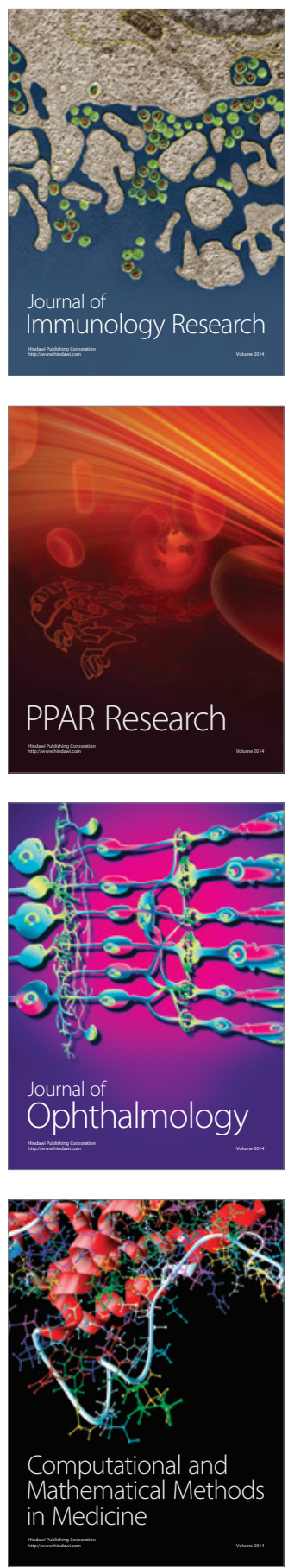

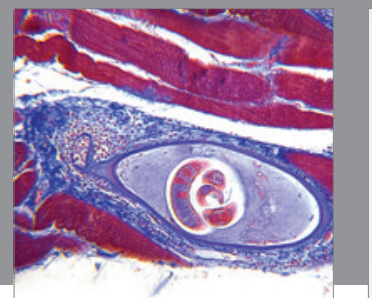

Gastroenterology

Research and Practice
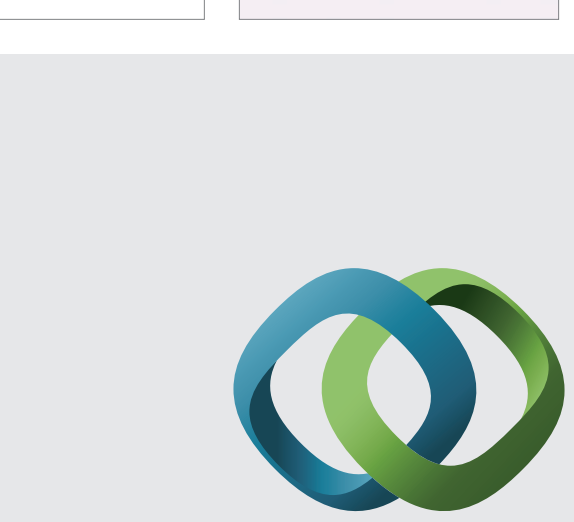

\section{Hindawi}

Submit your manuscripts at

http://www.hindawi.com
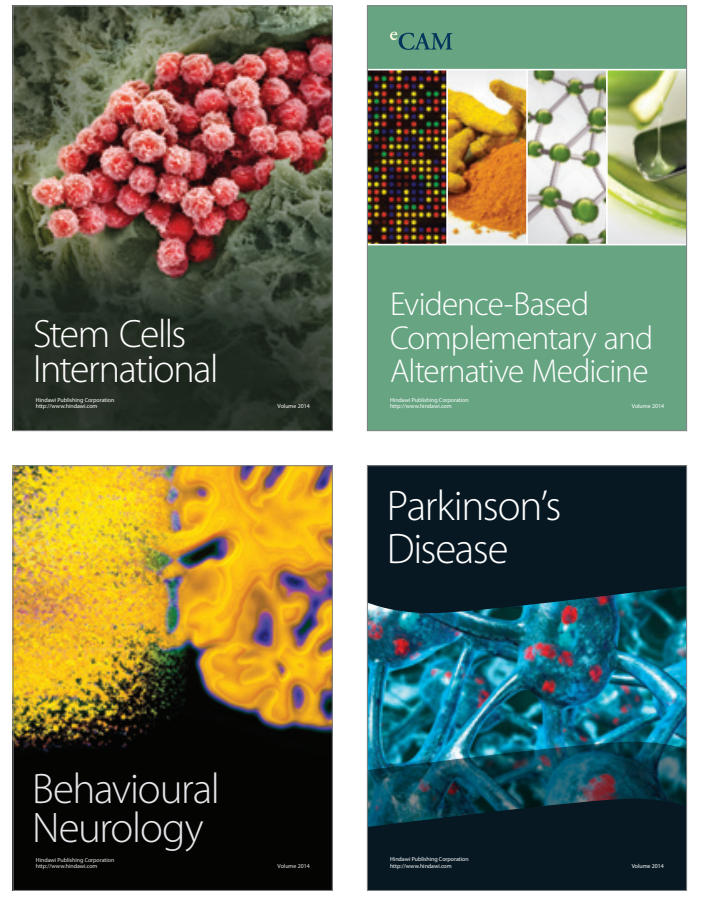
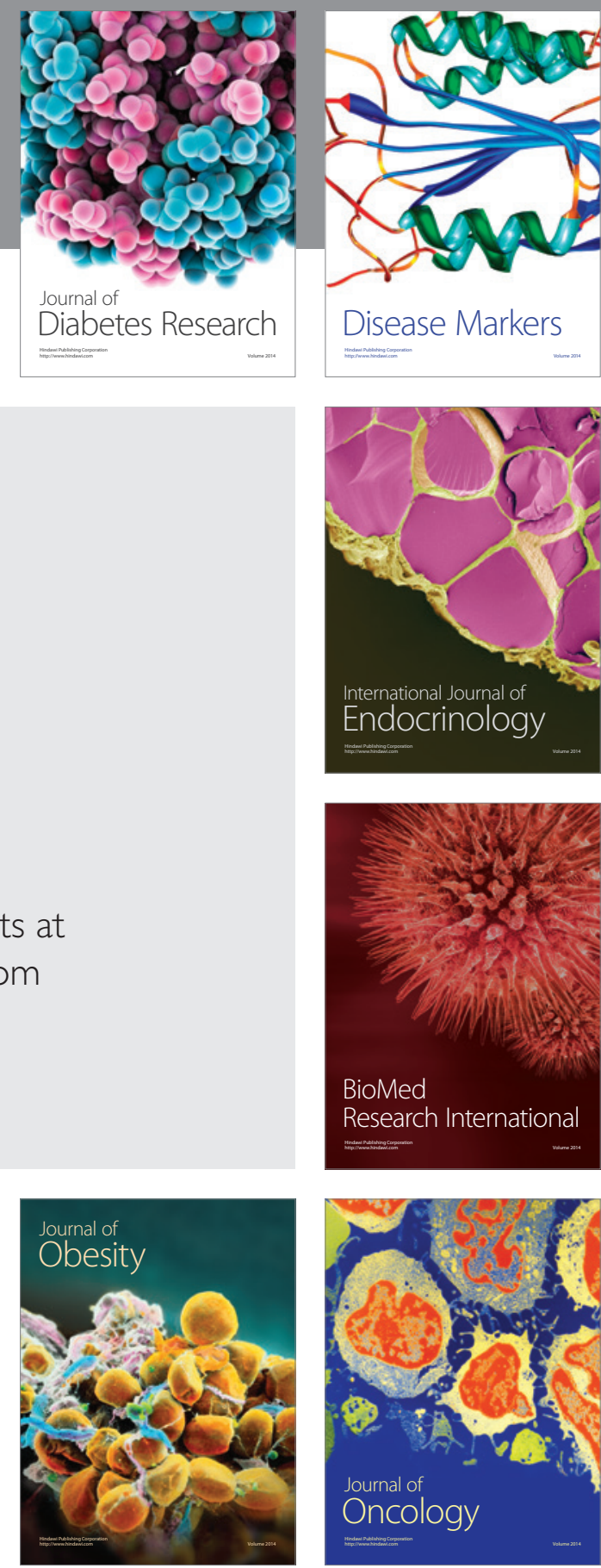

Disease Markers
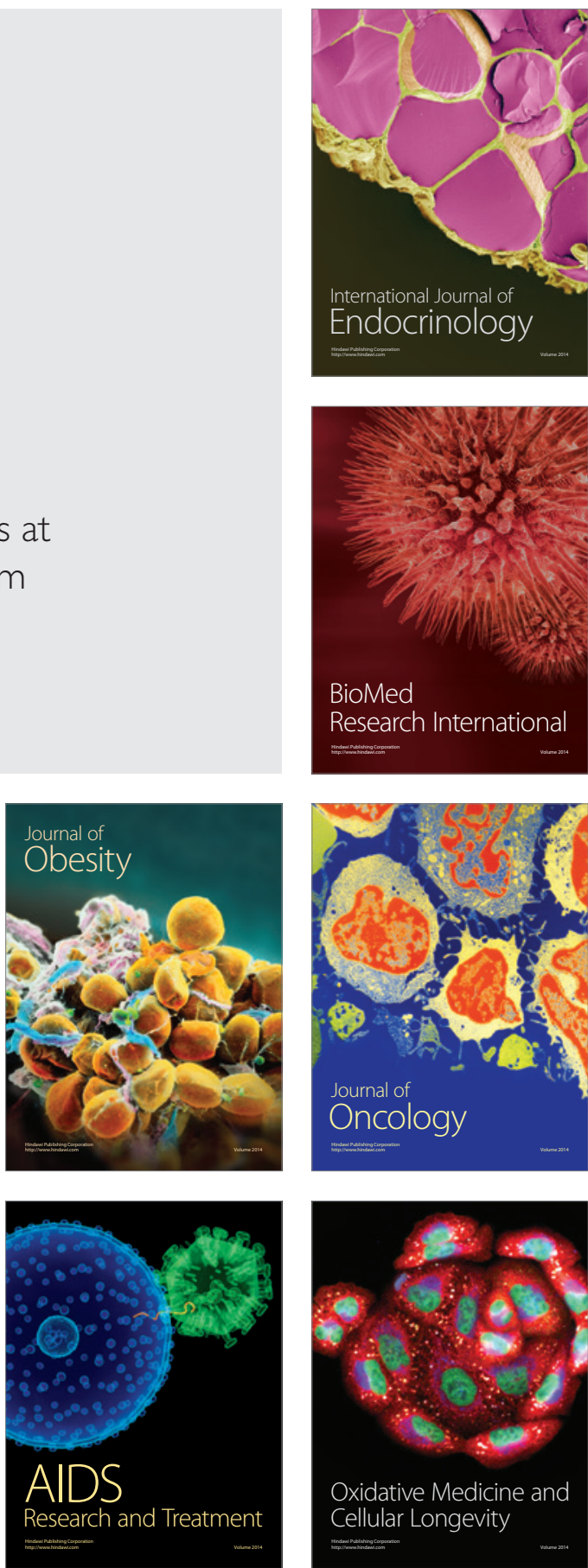